\title{
A wearable shear force transducer based on color spectrum analysis
}

\author{
Michael McGeehan 1,2, Michael Hahn 2, Salil Karipott ${ }^{2}$ and Keat Ghee Ong 2,* \\ 1 University of Oregon Phil and Penny Knight Campus for Accelerating Scientific Impact; mmcgeeha@uore- \\ gon.edu, karipott@uoregon.edu, kgong@uoregon.edu \\ 2 University of Oregon Department of Human Physiology; mmcgeeha@uoregon.edu, mhahn@uoregon.edu \\ * Correspondence: mmcgeeha@uoregon.edu
}

Simple Summary: There is growing need for wearable force sensors for healthcare and sport applications. For example, embedding a force sensor in the sole of a shoe could monitor shear (frictional) forces applied to the bottom of a foot and help prevent skin damage in susceptible populations, such as individuals with diabetes-related vascular dysfunction. Previous sensors for these applications have been limited due to being heavy and requiring large batteries. This paper presents the development and characterization of a novel sensor that measures shear forces based on changes in color light intensity. The sensor is comprised of a color pattern, which is exposed to an optical color sensor through a constrained viewing window. As the two sides of the sensor shear with respect to one another, different colors are exposed in the window, allowing the sensor to translate color variations to magnitude and direction of shear force. The sensor demonstrated a high degree of accuracy and repeatability for measuring shear forces in two directions. Compared to previous designs, the sensor is lighter weight and requires less power. As a demonstrative application, we present the design and implementation of a prototype wireless version of the sensor embedded in the sole of a shoe.

\begin{abstract}
The need for miniaturized shear force sensors is expanding, particularly for biomedical applications. Examples include measuring interfacial shear stresses between a human and an external device (e.g., footwear or a prosthesis). However, there are considerable challenges in designing a shear sensor for these applications due to the need for a small package, low power requirements, and resistance to interference from motion artifact and electromagnetic fields. This paper presents the design, fabrication, and characterization sensor that measures two-axis shear force by detecting displacement between a color panel and a red, green, and blue light-sensing photodiode. The sensor response to applied displacements and forces was characterized under benchtop testing conditions. We also present the design of a prototype wireless version of the sensor for integration into footwear. The sensor exhibited strong agreement with gold standard measurements for two axis shear displacements $\left(\mathrm{R}^{2}>0.99, \mathrm{RMSE} \leq 5.0 \mu \mathrm{m}\right)$ and forces $\left(\mathrm{R}^{2}>0.99, \mathrm{RMSE} \leq 0.94 \mathrm{~N}\right)$. This performance, along with the sensor's scalability, miniaturized form, and low power requirements make it well-suited a variety of biomedical applications.
\end{abstract}

Keywords: Sensor; optoelectronics; shear; force; biomechanics; gait; wearables; healthcare

\section{Graphical Abstract:}

\section{Introduction}

Miniaturized biosensors and wearable devices are increasingly popular tools for human health research, monitoring, and rehabilitation. There is a particular need for miniaturized tactile shear sensors integrated into exoskeletons, prostheses/orthoses, and footwear to evaluate human-device interface dynamics. In clinical settings, these sensors can be implemented to measure shear forces between a human skin/tissue and assistive device, helping to improve device design and optimize fit. Shear measurements are also critical for analyzing human gait biomechanics. For example, ground reaction shear forces 
are indicative of gait imbalance [1,2], thus continuous monitoring of these forces could help identify and mitigate fall risk. Excessive shear stress is also a leading factor in the formation of tissue ulcers at human-device interfaces (e.g., foot-shoe interface [3,4] and residual limb-prosthetic socket interface [5]). Integrating sensors at these locations could lead to improved management strategies to prevent ulcer formation, thus improving healthcare outcomes. In sport performance settings, measuring shearing between the plantar surface of the foot and shoe could lead to new innovations for evaluating and improving performance among athletes.

Despite the potential benefits, there remain considerable challenges in implementing miniaturized tactile shear sensors for biomedical and sport applications due to specific requirements and design constraints. Shear sensors designed for these use cases must be mechanically transparent to the user, and thus must adopt a small footprint or flexible form. The sensor should have low power requirements, allowing for wireless functionality. Designs should also be insusceptible to motion artifact or electromagnetic interference arising from human movement or nearby mechatronic systems. Various shear sensor designs have been reported, many of which are based on capacitive sensing principles [612]. However, capacitive sensing designs may be disadvantageous for biomedical and sport applications, as many of them require wired power sources or large batteries due high current draw $[7,8,10,13,14]$. Capacitive sensors may also be sensitive to electromagnetic interference and temperature changes $[6,7,10]$. In contrast, optical-based force transduction may be a better candidate for continuous force monitoring for biomedical applications due to low power requirements and relative insusceptibility to electromagnetic interference arising from the human body or mechatronic systems interacting with the sensor (e.g., powered assistive devices). Optoelectronic-based force transduction involves estimating force based on coupling of a light source (e.g., light-emitting diode (LED) $[15,16]$ or vertical cavity surface-emitting laser (VCSEL) [10-13]) and a light detector.

Optoelectronic sensing principles have been leveraged to measure both shear force and displacement [15-20]. McGeehan et al. (2021) [15] described a sensor for measuring two-axis shear force and displacement based on reflected intensities of red, green, and blue (RGB light). Briefly, this sensor measured shear strain based on optical coupling between an RGB LED and a photoresistor. The LED cycles red, green, and blue light, which is reflected off an adjacent surface displaying a $3 \times 3$ grid of red, green, blue, and magenta color panels. Only $1 / 9$ of the grid (corresponding the geometry of one color panel) is viewable at a time, constrained by a viewing window. Shearing between the adjacent surfaces reveals a different combination of color in the window to absorb and/or reflect RGB lights, allowing the photodiode to measure shear displacement based on relative changes in RGB light intensities. To measure shear force, an elastomer was adhered between the two surfaces [15].

The aforementioned sensing paradigm demonstrated promise, yet key limitations reduced the applicability for implementation in biomedical devices. One limitation was the increased microcontroller (MCU) processing required to control the timing of LED color cycling and sampling of the photoresistor. Similarly, the requirement to cycle the LED and sample from the photoresistor three times (red, green, and blue) for each force datapoint limited the overall sampling rate of the system. The purpose of this work was to design and test a variation of this sensing principle, whereby a white (full visible spectrum) LED is used to illuminate a color pattern. Reflected RGB intensity is measured via a photodiode with red, green, and blue filters as a method to measure shear force and displacement. This paper describes the design, fabrication, and characterization of a sensor that leverages this sensing paradigm. We also present the architecture and design of a prototype wireless version of the sensor integrated into the sole of a shoe for measuring interfacial shear force between the plantar surface of the foot and shoe. 


\section{Materials and Methods}

\subsection{Operational Principle}

The sensor measures multi-axial shear force and displacement based on optical coupling between white light from an LED reflected off a color pattern and a four-channel photodiode with red, green, blue, and white (full visible spectrum) sensing elements. Schematics of the sensor and sensing principle are shown in Figures 1 and 2. The instrumented side of the sensor has a windowed pattern (Figures 1 and 2, Surface A), whereas the opposite side of the sensor (Figures 1 and 2, Surface B) displays a colored grid consisting of green, red, blue, and magenta (red + blue) panels. Red, green, blue, and magenta color panels were designated for to their ability to selectively absorb or reflect colors corresponding to the photodiode's RGB-filtered sensing elements. Under resting conditions (i.e., zero strain), Surfaces A and B are perfectly aligned, revealing only green in the window on Surface A. In this configuration, the green surface in the window reflects primarily light at the green wavelength $(\sim 550 \mathrm{~nm})$ and absorbs red $(\sim 700 \mathrm{~nm})$ and blue $(\sim 450 \mathrm{~nm})$ wavelengths. This corresponding to higher magnitude green measurements at the photodiode, and lower magnitude red and blue measurements. Shearing between surfaces A and B causes a decrease in green reflective surface shown in the window on Surface A and an increase in either red (vertical shearing) or blue (horizontal shearing) (Figure 2). These changes are measured as variations in RGB light intensity (voltage changes) at the photodiode.



Figure 1. Expanded illustration of the sensor architecture and sensing paradigm. 1: Printed circuit board (PCB), 2: Sensor housing, 3: polyurethane elastomer (PUE) blocks, 4: Color pattern (Surface B), 5: Viewing window (Surface A), 6: LED, 7: Photodiode, 8: Emitted light, 9: Reflected light. 
Surface A: Window


No Shear

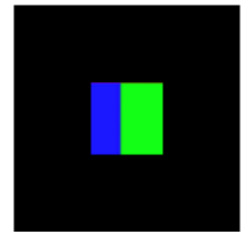

Horizontal Shear


Vertical Shear

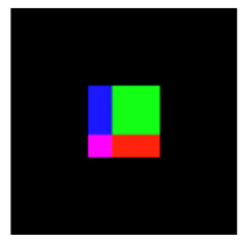

Diagonal Shear

Figure 2. Schematic showing relative displacement between Surfaces A and B under resting and shearing conditions.

The sensor is designed to be contactless, such that all electronic components are housed on one side. This configuration provides versatility in how the sensor is implemented for specific applications. For example, the pattern on Surface B could be printed on a 3Dprinted plate to make the sensor a standalone device (e.g., Section 2.2) or printed on the insole of a shoe (e.g., Section 2.3) to measure interfacial shear force between the sole of the foot and shoe.

\subsection{Sensor Architecture and Fabrication}

The sensor is comprised of three main components: a) A 3D-printed housing for the electronics, which limits the field of view of the photodiode via a $10 \times 10 \mathrm{~mm}$ black trim window (Surface A), b) a $3 \times 3$ color grid composed of red, green, blue, and magenta color panels, and c) an intermediate layer polyurethane elastomer (PUE) blocks separating Surfaces A and B (Figure 1). The sensor packaging, including circuitry is $34 \times 27 \times 7 \mathrm{~mm}$ (thickness) and weights $0.81 \mathrm{~g}$. The thickness of the sensor is largely dependent upon the thickness of the elastomer layer. The maximum range and sensitivity towards shear stress can be tuned by changing the elastomer material and thickness, which affects the stressstrain properties of the material [21]. For example, the PUE used in this sensor configuration has an elastic modulus of $4.0 \mathrm{MPa}$. Alternatively, a polydimethylsiloxane (PDMS) elastomer with a lower elastic modulus $(250-450 \mathrm{kPa}[20,22])$ could be used for higher resolution, lower range sensor configuration.

The LED (Everlight Electronics, 45-21/LK2C-B38452C4CB2/2T) is $3.0 \times 2.0 \times 1.4 \mathrm{~mm}$ (thickness) and emits light in a 400-800 nm spectrum. At $20 \mathrm{~mA}$, the LED has a brightness of 2,000 milicandella (mcd). The photodiode (Texas Advanced Optoelectronic Solutions, TCS34725) is $2.0 \times 2.4 \times 1.6 \mathrm{~mm}$. The photodiode has individual red, green, blue, and clear light sensing elements with an infrared blocking filter. Four integrated analog-to-digital converters (ADC) convert photodiode currents to 16-bit digital values. Peak responsivity of the sensing elements occurs at $620 \mathrm{~nm}$ (red), $550 \mathrm{~nm}$ (green), and $475 \mathrm{~nm}$ (blue). The device utilizes an $\mathrm{I}^{2} \mathrm{C}$ fast communication protocol for data rates of up to $400 \mathrm{kbit} / \mathrm{s}$. It consumes $2.5 \mu \mathrm{A}$ in standby mode and $65 \mu \mathrm{A}$ when operating.

The LED and photodiode are soldered to a printed circuit board (PCB) (OshPark Inc., $2 \mathrm{oz}$ Copper, $0.8 \mathrm{~mm}$ thickness), which is housed in a 3D printed methylacrylate photopolymer resin (FormLabs Inc., Rigid 10k). This resin has a tensile modulus of $10 \mathrm{GPa}$ and a flexural modulus of $9 \mathrm{GPa}$. By employing rapid prototyping technology in the 
fabrication process, the sensor's cost-efficiency and versatility are improved. The color pattern was printed on an adhesive-backed vinyl with matte finish (Strathmore Inc., 59635).

To provide mechanical elasticity to the sensor, a flexible PUE layer was incorporated between Surfaces A and B. The PUE (durometer: $80 \mathrm{a}$, thickness: $3.5 \mathrm{~mm}$ ) was laser cut into 4 cuboids $(8.0 \times 8.0 \times 3.5$ (thickness) $\mathrm{mm}$ ) adhered between the surfaces at each corner of the surface interface (Loctite 401, Düsseldorf, Germany) (Figure 1). The glue was allowed to cure for $24 \mathrm{~h}$ at room temperature. The PUE has a reported elastic modulus ranging 4.7 $-7.4 \mathrm{MPa}[23]$.

\subsection{Prototype Wireless Sensor System and Integration Into Footwear For Human Gait Monitoring}

A prototype wireless configuration of this sensor was also designed with the goal of integration into footwear for human gait monitoring (Figure 3). For this design, a Bluetooth Low-Energy (BLE) transceiver (Silicon Labs Inc., BLE113) with an integrated microcontroller unit (MCU) was incorporated to control the electronics and wirelessly stream data to a computer or smartphone. A CR2016 lithium manganese dioxide coin cell battery $(3 \mathrm{~V}, 90 \mathrm{mAh})$ was also included to provide wireless power to the system. A battery of this capacity can power the system during active data collection for an estimated $3 \mathrm{~h}$. A higher capacity battery could be incorporated in the future to lengthen collection time. In this configuration, the sensor packaging is $34 \times 27 \times 9 \mathrm{~mm}$ (thickness) and weighs $1.16 \mathrm{~g}$ including the battery.

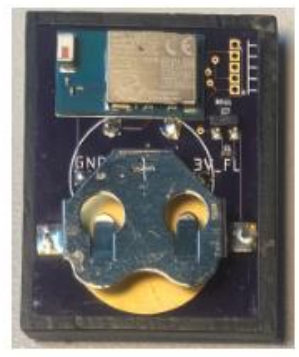

(A)

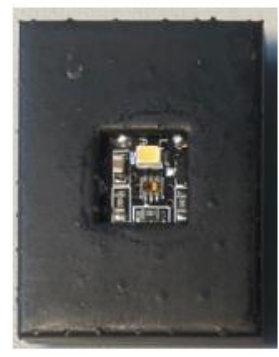

(B)

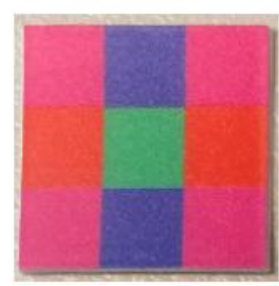

(C)



(D)

Figure 3. Photos of wireless sensor implementation. (A) Back of instrumented side of sensor (PCB with BLE113 module and coin cell battery clip), (B) Front of instrumented side of sensor (Surface A), (C) Color pattern on underside of insole, (D) Wireless sensor embedded in midsole of shoe (seen through excised viewing window in fabric).

The BLE113 module contains an MCU for controlling the sensor, local memory for hosting data and end user applications, and a BLE radio for bi-directional communication with a computer or smartphone. The MCU can set the device in two operation modes: standby/sleep mode and active sensing mode. In standby mode, the sensor receives no power and the BLE113 consumes only $0.4 \mu \mathrm{A}$. In active sensing mode where the LED and 
photodiode are active and data are being streamed to an external Bluetooth-enabled device, the BLE113 consumes $18 \mathrm{~mA}$. A block diagram of the telemetry platform is depicted in Figure 4. The BLE113 acts as the host device and sets the sensing mode based on client inputs. In active mode, the LED is turned on and photodiode sampling is prompted via an $\mathrm{I}^{2} \mathrm{C}$-fast communication protocol. A program onboard the $\mathrm{MCU}$ stores photodiode measurements and creates a 20-byte data packet. These packets can be transmitted to the client device (a computer) at a rate of $30 \mathrm{~Hz}$, giving the sensor an effective sampling rate of $180 \mathrm{~Hz}$ and providing near real-time data streaming to the end user.

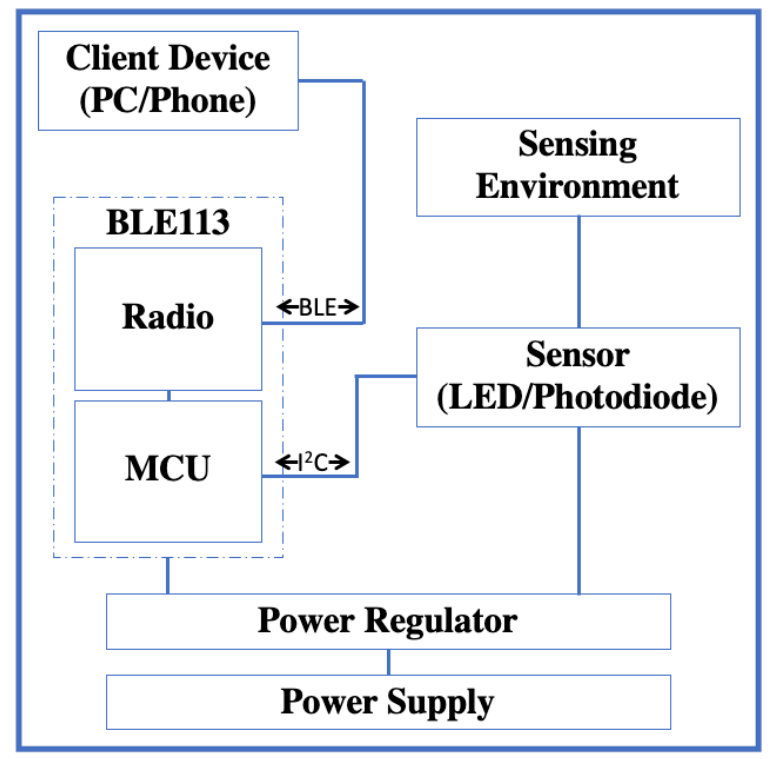

Figure 4. Block diagram of the telemetry platform.

For this application Surface B was adhered to the underside of the shoe insole and Surface A was embedded into the midsole of the shoe. To integrate the sensor into the shoe, a rectangular hole $(\mathrm{L} \times \mathrm{W} \times \mathrm{H})$ matching the dimensions of Surface A was excised from the midsole (Figure 3). The instrumented side of the sensor was then secured in the midsole (Loctite 401, Düsseldorf, Germany). The color patten (Surface B) was adhered to the corresponding location on the underside of the shoe's insole. The PUE elastomer layer was then adhered between the two surfaces using the methods described in Section 2.2.

\subsection{Sensor Characterization}

The sensor's response to both displacement and applied shear force were measured. Measuring the response to displacement serves to establish the baseline sensitivity of the sensor towards change in strain. Measuring the response to shear force is useful to further characterize the sensor's performance under the ultimate use conditions.

For displacement testing, a $3 \mathrm{~mm}$-thick 3D printed spacer was inserted in the place of the PUE. To apply controlled displacements (i.e., shear strains), a 3D printed fixture was designed to secure the sensor to the machining platform of a modified computed numeric control (CNC) router (FoxAlien, 3018-SE V2) (Figure 5a). A second 3D printed fixture displaying the color pattern was affixed in the CNC spindle bracket to allow controlled displacement between sensor Surfaces A and B. The CNC's stepper motors (NEMA 17) allow for controlled displacement with an accuracy of $\pm 10 \mu \mathrm{m}$. Surface $B$ was then 
displaced in $200 \mu \mathrm{m}$ increments for a range of $0-2000 \mu \mathrm{m}$ in the $\mathrm{X}$ and $\mathrm{Y}$ planes (parallel to the surface plane of the sensor), as illustrated in Figure 4a. At each $200 \mu \mathrm{m}$ position, static measurements of red, green, blue, and clear (full spectrum) color intensity were recorded. To characterize the repeatability of these measurements, a total of 10 measurements were recorded at each position and inter-measurement variability was calculated. In total, the sensor response was characterized at 121 locations (1210 datapoints). Data from the sensor were compared to position data from the CNC.



(A)



(B)

Figure 5. (A) Testing configuration for the CNC mill and (B) mechanical testing system (MTS). 1: Milling platform, 2: Sensor, 3: Sensor fixture for attaching to milling base, 4: Spindle bracket, 5: Spindle adapter displaying color pattern, 6: Stepper motor, 7: MTS, 8: Sensor Surface A, 9: Sensors Surface B, 10: Printed circuit board (PCB), 11: Sensor fixture for attaching to MTS, 12: Holes for set screws, 13: Load cell

To characterize the sensor's response to shear force, a 3D-printed fixture was designed to secure the sensor components in a mechanical testing system (MTS) (EnduraTEC ELF, TA Instruments, New Castle, DE). Using different equipment to characterize sensor force and displacement measurements was necessary because the CNC used in displacement testing was not able to measure force. The MTS was actuated for loads ranging 0$100 \mathrm{~N}$ in $10 \mathrm{~N}$ increments, measured by a load cell (TA Instruments, 1516FQG-100) placed in series with the testing fixture (Figure $5 b$ ). The maximal load was dictated by the maximum loading capacity of the MTS. At each loading level, a static measurement of red, green, blue, and clear (full spectrum) color intensity was recorded. To characterize the repeatability of these measurements, a total of 10 measurements were recorded at each position and inter-measurement variability was calculated. Sensor-derived measurements of shear stress were then compared to data from the load cell. Hysteresis of the sensor was also characterized by loading and unloading the sensor $0-100 \mathrm{~N}$ with a $0.1 \mathrm{~Hz}$ duty cycle. To best represent real-world conditions, all data were collected under ambient light conditions in the laboratory. 
Displacement data from the CNC (accuracy: $\pm 10 \mu \mathrm{m}$ ) and load cell data from the mechanical testing system (accuracy: $\pm 0.0001 \mathrm{~N}$ ) were used as gold standard comparator values to model and characterize sensor performance. The four digital values for red, green, blue, and clear intensity (outputs from the photodiode) were used as variables to model the sensor's response to shear displacement/force. Model fits for both displacement and force were derived for the sensor's response (i.e., RGB light intensities) compared to the gold standard value.

Exponential Gaussian Process (GP) regression was used to model the sensor response for both displacement and shear force conditions (Mathworks, Natick, MA). Compared to traditional regression models, GPs are advantageous because the user may add a priori knowledge and specifications about the shape and behavior of the model by selecting different kernel functions (e.g., linear vs. exponential). Five rounds of cross-validation were performed using randomized data partitions. The validation results were averaged across the rounds to give an overall characterization of the model's predictive performance. Sensor performance was characterized using coefficient of determination $\left(\mathrm{R}^{2}\right)$, mean absolute error (MAE), and root-mean-squared error (RMSE) values across the full range of conditions tested.

\section{Results}

Sensor-derived measurements of displacement demonstrated a high level of agreement with gold standard data. In the vertical direction, the modeled sensor data exhibited an $\mathrm{R}^{2}>0.99$ with an RMSE of $4.5 \mu \mathrm{m}$ (Table 1, Figure 6). The largest residuals occurred for displacements greater than $1 \mathrm{~mm}$, with the highest error value $(40 \mu \mathrm{m})$ occurring at 1.8 $\mathrm{mm}$ displacement (Figure 5). Similar performance was observed in the horizontal direction $\left(\mathrm{R}^{2}>0.99\right.$, RMSE: $\left.5.0 \mu \mathrm{m}\right)$. In this direction, the residuals were relatively consistent across the full range of tested displacements, with the highest residual (50 $\mu \mathrm{m})$ occurring at $0.6 \mathrm{~mm}$ displacement (Figure 5).

Table 1. Summary of sensor performance.

\begin{tabular}{rccll}
\hline & \multicolumn{2}{c}{ Displacement $(\boldsymbol{\mu m})$} & \multicolumn{2}{c}{ Force $(\mathbf{N})$} \\
& Vertical & Horizontal & Vertical & Horizontal \\
\hline $\mathbf{R}^{\mathbf{2}}$ & $>0.99$ & $>0.99$ & $>0.99$ & $>0.99$ \\
RMSE & 4.5 & 5.0 & 0.94 & 0.84 \\
MAE & 1.7 & 1.8 & 0.31 & 0.54 \\
\hline
\end{tabular}



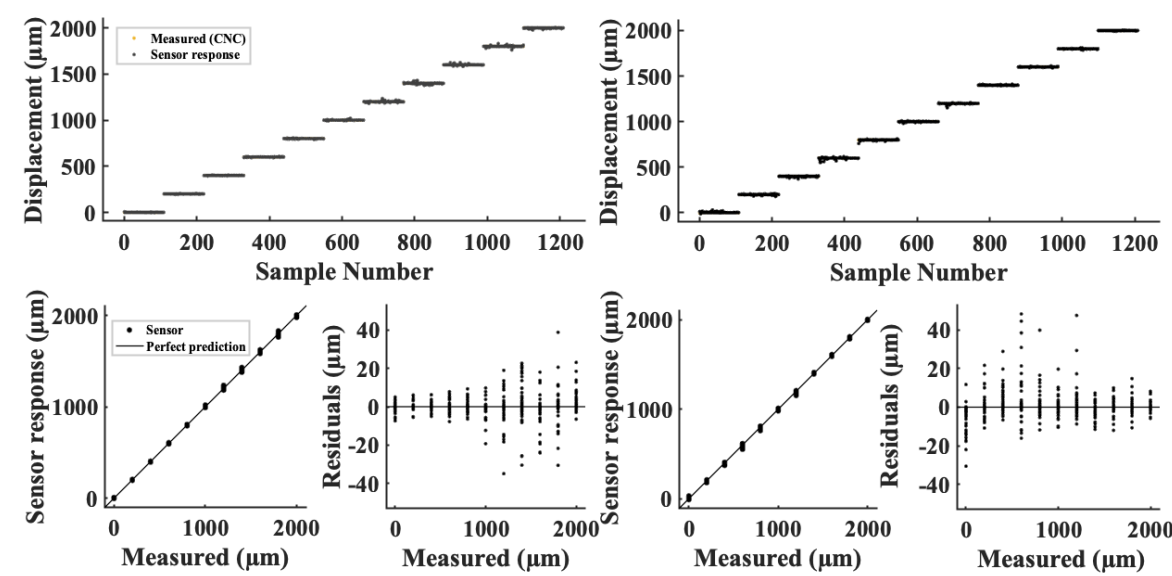

Figure 6. Sensor performance compared to gold standard displacement measurements in the vertical (left) and horizontal (right) directions. $n=1201$.

Sensor-derived measurements of shear force also exhibited good agreement with gold standard load cell data (Table 1, Figure 7). In the vertical direction, the modeled sensor data were strongly correlated with the load cell ( ${ }^{2}>0.99$, RMSE: 0.94 N, MAE: 0.31 $\mathrm{N}$ ) across the $0-100 \mathrm{~N}$ loading range. Similar performance was evident in the horizonal direction ( $\mathrm{R}^{2}>0.99$, RMSE: $0.84 \mathrm{~N}$, MAE: $0.54 \mathrm{~N}$ ). The highest residuals (i.e., error) occurred in the $40-70 \mathrm{~N}$ loading range for vertical forces and were relatively evenly distributed across the full loading range of horizonal forces.


Figure 7. Sensor performance compared to gold standard displacement measurements in the vertical (left) and horizontal (right) directions. $n=121$.

To investigate the elastic behavior of the physical sensor package (i.e., PUE, assuming the resin housing is rigid) the stiffness and hysteresis of the packaged sensor were evaluated. The sensor exhibited a linear relationship between load and displacement $\left(R^{2}\right.$ $>0.98$ ) as measured via the load cell and MTS displacement sensor. Average hysteresis was $7.8 \mathrm{~N}$ across the full range of loads and displacements (Figure 8). The relationship between force $(\mathrm{F})$ and displacement $(x)$ was $101.0 \mathrm{~N} / \mathrm{mm}$. Assuming rigidity of the sensor housing, the shear modulus of the PUE with surface area " $\mathrm{A}$ " was calculated to be 3.95 $\operatorname{MPa}$ (eq. 1). 
$G=\frac{F / x}{A}=\frac{101 \mathrm{~N} / \mathrm{mm}}{25.6 \mathrm{~mm}^{2}}=3.95 \mathrm{MPa}$



Figure 8. Hysteresis response for sensor measured via MTS and load cell.

\section{Discussion}

The goal of this work was to evaluate the ability of a color sensing paradigm based on optical coupling between a white light LED and an RGB photodiode to detect two-axis applied shear displacement and force. We presented the design, fabrication, and characterization of a simple and low-cost sensor based on this sensing paradigm. Overall, the sensor demonstrated a robust ability to detect shear displacement within a $2 \mathrm{~mm}$ dynamic range and applied shear force within a $100 \mathrm{~N}$ dynamic range. The performance of the sensor, combined with its small form and scalable design support its use for a variety of biomedical and sport applications. As a demonstrative implementation, we presented a prototype wireless version of the sensor integrated into the sole of the shoe. Future work will investigate the efficacy of this design to monitor human gait biomechanics.

The sensor demonstrated a high level of accuracy and resolution for measuring twoaxis shear displacement and applied forces under benchtop testing conditions. Average inter-measurement variability was $<0.1 \%$ for all conditions, indicating that the sensor is repeatably accurate. The sensor exhibited linearity of $>0.99$ for displacement and $>0.98$ for forces, as calculated by the slope of the relationship between the sensor output and gold standard values. High linearity is beneficial for wearable wireless sensors, because it potentially allows for simplified signal conditioning and data processing, thereby reducing the computational requirements for an MCU.

The highest displacement error was $50 \mu \mathrm{m}(2.5 \%)$ for displacement, whereas the highest error for applied force was $5.2 \mathrm{~N}(5.2 \%)$. The relatively higher error for force sensing may be attributed to minor imperfections in the sensor fabrication process. For example, the PUE layers and their interfaces with Surfaces A and B may not have been 
completely homogenous, resulting in small inconsistencies in stiffness across the loading range. Similarly, error could occur due to slippage of the glue adhering the PUE between Surfaces A and B. Future designs could improve the adhesion process or utilize techniques such as injection molding to directly incorporate the PUE layer into the sensor structure.

The sensor exhibited an average stiffness of $101 \mathrm{~N} / \mathrm{mm}$ and hysteresis of $7.8 \mathrm{~N}$ across a $0-100 \mathrm{~N}$ loading range. The sensor stiffness derived from mechanical testing was slightly lower than previously reported material properties for PUE [23]. This discrepancy may indicate that the sensor housing is not completely rigid under the testing loads or that small amounts of slippage of the glue occurred during testing. The hysteresis could be a limitation for sensing shear forces in biomechanical applications such as human gait, where loading and unloading characteristics are both important considerations [24]. Sensor hysteresis could be improved by incorporating other elastomers such as PDMS, which was previously shown to have less hysteresis in similarly configured sensors [12,15]. Other elastic structures such as coil and/or leaf springs could also be used $[9,25]$.

Anterior-posterior and medial-lateral shear forces occurring during level ground human gait range $0-1.5 \mathrm{~N} / \mathrm{kg}$ of body weight [26]. For a $70 \mathrm{~kg}$ individual, this equates to 0 $-105 \mathrm{~N}$, indicating that the sensor in its current configuration is well-suited for monitoring shear forces under these conditions. Future work will evaluate the use of the prototype in-shoe sensor (Section 2.3) for measuring foot-shoe interface dynamics during walking. Excessive shear forces are a leading factor in tissue damage on the plantar surface of the foot $[3,4]$. This sensor could serve as a monitoring system for tissue health and lead to improved prevention and healthcare strategies for plantar tissue ulcers. Shear forces may increase as high as $4 \mathrm{~N} / \mathrm{kg}$ in other walking conditions (e.g., stair ascent/descent and ramp ascent/descent [26]) and higher in athletics scenarios [27-29]. Future research should explore the ability of the current sensor design to measure forces in this range. This sensor was also designed to be scalable to for use in different sensing applications with different range and accuracy requirements. Future research will evaluate the use of different design variations such as use of elastomers with different elastic moduli to custom tune the sensor for specific applications. For example, a PDMS elastomer layer with a lower elastic modulus $(250-450 \mathrm{kPa}[20,22])$ could be used for a higher resolution, lower range sensor configuration.

In addition to scalability, this sensor offers several other advantages compared to previous shear sensor designs. The use of rapid fabrication techniques such as 3D printing and use of PCBs is advantageous for quickly iterating through the design modification and testing process. However, the efficacy of traditional mass fabrication techniques such as injection molding and automated circuit population should be explored, as they may necessary to ensure repeatable and reliable mass fabrication.

The data demonstrate good differentiation between horizontal and vertical shearing and indicate that the sensor performs equally well in both directions (Table1, Figures 6 and 7). Historically, the ability to measure multi-axial shear displacement and force has been primarily limited to use of strain gauges [10,30]. Compared to this sensor, other wearable force sensors have been heavier (e.g., $375 \mathrm{~g}$ [30]), require more power (e.g., 700 $\mathrm{mA}$ [7]), and/or need wired power sources [31-36]. This sensor has a current draw of $\sim 21$ $\mathrm{mA}$ without the BLE module and $\sim 30 \mathrm{~mA}$ with the BLE module, allowing wireless operation and minimal battery requirements.

\section{Conclusions}

This work demonstrated efficacy of an optical-based sensor for measuring two-axis shear displacement and force. We presented the design, fabrication, and characterization of the sensor under benchtop conditions. A prototype wireless design integrated into the 
midsole of a shoe was also presented. Overall, the sensor exhibited accurate and repeatable measurements of two-axis shearing. This technology is comparatively advantageous to previous wearable shear sensor designs, due to its small mass and lower power requirements. These properties, along with its small, low-cost, and scalable design support the use of this sensor for a variety of biomedical applications.

\section{Patents}

This work is part of United States Provisional Patent Pending No.: 63/172,615 


\section{Supplementary Materials: N/A}

Author Contributions: MAM was responsible for the design, fabrication, and experimental testing of the sensor. He carried out all sensor testing, data analysis, and manuscript preparation. SSL assisted with the development of the sensing principle as well as sensor design and fabrication. He also assisted with manuscript preparation. MEH was involved in the conceptual design of the sensor and helped design the experimental testing protocols. He also assisted with designing statistical and data analysis methods and manuscript preparation. KGO was responsible for overseeing the entirety of sensor design and testing. He co-developed the sensing principle, helped design testing protocols, and assisted with manuscript preparation.

Funding: This work was supported by the Joe and Clara Tsai Foundation and the Wu Tsai Human Performance Alliance at Oregon, Knight Campus for Accelerating Scientific Impact.

Institutional Review Board Statement: This work was not required to undergo IRB review. No human subjects were required for this work, thus no informed consent was necessary.

Informed Consent Statement: This work was not required to undergo IRB review. No human subjects were required for this work, thus no informed consent was necessary.

Data Availability Statement: All data pertaining to this work will be make available upon request to the authors.

\section{Acknowledgments: N/A}

Conflicts of Interest: The authors declare no conflicts of interest for this work.

\section{References}

1. Jiang, S.; Zhang, B.; Wei, D. The elderly fall risk assessment and prediction based on gait analysis. Proc. - 11th IEEE Int. Conf. Comput. Inf. Technol. CIT 2011 2011, 176-180, doi:10.1109/CIT.2011.82.

2. Hosein, R.; Lord, M. A study of in-shoe plantar shear in normals. Clin. Biomech. 2000, 15, 46-53, doi:10.1016/S02680033(98)00059-X.

3. Jones, A.D.; De Siqueira, J.; Nixon, J.E.; Siddle, H.J.; Culmer, P.R.; Russell, D.A. Plantar shear stress in the diabetic foot: A systematic review and meta-analysis. Diabet. Med. 2022, 39, doi:10.1111/DME.14661.

4. Yavuz, M.; Ersen, A.; Hartos, J.; Schwarz, B.; Garrett, A.G.; Lavery, L.A.; Wukich, D.K.; Adams, L.S. Plantar Shear Stress in Individuals With a History of Diabetic Foot Ulcer: An Emerging Predictive Marker for Foot Ulceration. Diabetes Care 2017, 40, e14, doi:10.2337/DC16-2204.

5. Zhang, M.; Turner-Smith, A.R.; Roberts, V.C.; Tanner, A. Frictional action at lower limb/prosthetic socket interface. In Proceedings of the Medical Engineering and Physics; Butterworth-Heinemann Ltd, 1996; Vol. 18, pp. 207-214.

6. Cheng, M.-Y.; Lin, C.-L.; Lai, Y.-T.; Yang, Y.-J. A Polymer-Based Capacitive Sensing Array for Normal and Shear Force Measurement. Sensors 2010, 10, 10211-10225, doi:10.3390/s101110211.

7. Laszczak, P.; Jiang, L.; Bader, D.L.; Moser, D.; Zahedi, S. Development and validation of a 3D-printed interfacial stress sensor for prosthetic applications. Med. Eng. Phys. 2015, 37, 132-137, doi:10.1016/j.medengphy.2014.10.002.

8. Laszczak, P.; McGrath, M.; Tang, J.; Gao, J.; Jiang, L.; Bader, D.L.; Moser, D.; Zahedi, S. A pressure and shear sensor system for stress measurement at lower limb residuum/socket interface. Med. Eng. Phys. 2016, 38, 695-700, doi:10.1016/j.medengphy.2016.04.007.

9. Brookhuis, R.A.; Droogendijk, H.; de Boer, M.J.; Sanders, R.G.P.; Lammerink, T.S.J.; Wiegerink, R.J.; Krijnen, G.J.M. Six-axis force-torque sensor with a large range for biomechanical applications. J. Micromechanics Microengineering 2014, 24, 035015, doi:10.1088/0960-1317/24/3/035015.

10. Sanders, J.E.; Daly, C.H. Measurement of Stresses in Three Orthogonal Directions at the Residual Limb-Prosthetic Socket Interface. IEEE Trans. Rehabil. Eng. 1993, 1, 79-85, doi:10.1109/86.242421.

11. Sanders, J.E.; Daly, C.H.; Burgess, E.M. Interface shear stresses during ambulation with a below-knee prosthetic limb. J. Rehabil. Res. Dev. 1992, 29, 1-8, doi:10.1682/jrrd.1992.10.0001.

12. Choi, E.; Hwang, S.; Yoon, Y.; Seo, H.; Lee, J.; Yeom, S.; Ryu, G.; Yang, H.; Kim, S.; Sul, O.; et al. Highly sensitive tactile shear sensor using spatially digitized contact electrodes. Sensors (Switzerland) 2019, 19, doi:10.3390/s19061300. 
13. Sanders, J.E.; Zachariah, S.G.; Baker, A.B.; Greve, J.M.; Clinton, C. Effects of changes in cadence, prosthetic componentry, and time on interface pressures and shear stresses of three trans-tibial amputees. Clin. Biomech. 2000, 15, 684-694.

14. Laszczak, P.; Mcgrath, M.; Tang, J.; Gao, J.; Jiang, L.; Bader, D.L.; Moser, D.; Zahedi, S. A pressure and shear sensor system for stress measurement at lower limb residuum/socket interface. Med. Eng. Phys. 2016, 38, 695-700, doi:10.1016/j.medengphy.2016.04.007.

15. McGeehan, M.A.; Karipott, S.S.; Hahn, M.E.; Morgenroth, D.C.; Ong, K.G. An Optoelectronics-Based Sensor for Measuring Multi-Axial Shear Stresses. IEEE Sens. J. 2021, 1-1, doi:10.1109/JSEN.2021.3117935.

16. McGeehan, M.A.; Hahn, M.E.; Karipott, S.S.; Shuaib, M.; Ong, K.G. Optical-Based Sensing of Shear Strain using Reflective Color Patterns. Sensors Actuators A Phys. 2022, 113372, doi:10.1016/J.SNA.2022.113372.

17. Missinne, J.; Bosman, E.; Van Hoe, B.; Van Steenberge, G.; Kalathimekkad, S.; Van Daele, P.; Vanfleteren, J. Flexible shear sensor based on embedded optoelectronic components. IEEE Photonics Technol. Lett. 2011, 23, 771-773, doi:10.1109/LPT.2011.2134844.

18. Missinne, J.; Bosman, E.; Van Hoe, B.; Van Steenberge, G.; Van Daele, P.; Vanfleteren, J. Embedded flexible optical shear sensor. Proc. IEEE Sensors 2010, 987-990, doi:10.1109/ICSENS.2010.5690919.

19. Missinne, J.; Bosman, E.; Van Hoe, B.; Verplancke, R.; Van Steenberge, G.; Kalathimekkad, S.; Van Daele, P.; Vanfleteren, J. Two axis optoelectronic tactile shear stress sensor. Sensors Actuators, A Phys. 2012, 186, 63-68, doi:10.1016/j.sna.2012.01.038.

20. Missinne, J.; Bosman, E.; Van Hoe, B.; Verplancke, R.; Van Steenberge, G.; Kalathimekkad, S.; Van Daele, P.; Jan, V. Ultra thin optical tactile shear sensor. Procedia Eng. 2011, 25, 1393-1396.

21. Nunes, L.C.S. Shear modulus estimation of the polymer polydimethylsiloxane (PDMS) using digital image correlation. Mater. Des. 2010, 31, 583-588, doi:10.1016/j.matdes.2009.07.012.

Lötters, J.C.; Olthuis, W.; Veltink, P.H.; Bergveld, P. The mechanical properties of the rubber elastic polymer polydimethylsiloxane for sensor applications. J. Micromechanics Microengineering 1997, 7, 145-147, doi:10.1088/0960-1317/7/3/017.

23. Kanyanta, V.; Ivankovic, A. Mechanical characterisation of polyurethane elastomer for biomedical applications. J. Mech. Behav. Biomed. Mater. 2010, 3, 51-62, doi:10.1016/J.JMBBM.2009.03.005.

24. Jaeger, R.J.; Vanitchatchavan, P. Ground reaction forces during termination of human gait. J. Biomech. 1992, 25, 1233-1236, doi:10.1016/0021-9290(92)90080-K.

25. Osada, M.; Ito, N.; Nakanishi, Y.; Inaba, M. Realization of flexible motion by musculoskeletal humanoid “Kojiro" with addon nonlinear spring units. 2010 10th IEEE-RAS Int. Conf. Humanoid Robot. Humanoids 2010 2010, 174-179, doi:10.1109/ICHR.2010.5686271.

26. Redfern, M.S.; Cham, R.; Gielo-Perczak, K.; Grönqvist, R.; Hirvonen, M.; Lanshammar, H.; Marpet, M.; Pai, C.Y.C.; Powers, C. Biomechanics of slips. Ergonomics 2001, 44, 1138-1166, doi:10.1080/00140130110085547.

27. Yu, L.; Mei, Q.; Xiang, L.; Liu, W.; Mohamad, N.I.; István, B.; Fernandez, J.; Gu, Y. Principal Component Analysis of the Running Ground Reaction Forces With Different Speeds. Front. Bioeng. Biotechnol. 2021, 9, 205, doi:10.3389/FBIOE.2021.629809/BIBTEX.

28. Nilsson, J.; Thorstensson, A. Ground reaction forces at different speeds of human walking and running. Acta Physiol. Scand. 1989, 136, 217-227, doi:10.1111/J.1748-1716.1989.TB08655.X.

29. de Camargo Neves Sacco, I.; Takahashi, H.Y.; Suda, E.Y.; Battistella, L.R.; Kavamoto, C.A.; Fernandes Lopes, J.A.; de Vasconcelos, J.C.P. Ground reaction force in basketball cutting maneuvers with and without ankle bracing and taping. Sao Paulo Med. J. 2006, 124, 245-252, doi:10.1590/S1516-31802006000500002.

30. Sanders, J.E.; Daly, C.H.; Burgess, E.M. Interface shear stresses during ambulation with a below-knee prosthetic limb. J. Rehabil. Res. Dev. 1992, 29, 1-8, doi:10.1682/jrrd.1992.10.0001.

31. Courtney, A.; Orendurff, M.S.; Buis, A. Effect of alignment perturbations in a trans-tibial prosthesis user: A pilot study. J. Rehabil. Med. 2016, 48, 396-401, doi:10.2340/16501977-2075.

32. Safari, M.R.; Tafti, N.; Aminian, G. Socket Interface Pressure and Amputee Reported Outcomes for Comfortable and Uncomfortable Conditions of Patellar Tendon Bearing Socket: A Pilot Study. Assist. Technol. 2015, 27, 24-31, doi:10.1080/10400435.2014.949016.

33. Schiff, A.; Havey, R.; Carandang, G.; Wickman, A.; Angelico, J.; Patwardhan, A.; Pinzur, M. Quantification of Shear Stresses Within a Transtibial Prosthetic Socket. Foot ankle Int. 2014, 35, 779-782, doi:10.1177/1071100714535201.

34. Gholizadeh, H.; Azuan, N.; Osman, A.; Eshraghi, A.; Anuar, N.; Razak, A. Clinical implication of interface pressure for a new prosthetic suspension system. Biomed. Eng. Online 2014, 13, doi:10.1186/1475-925X-13-89.

35. Ali, S.; Abu Osman, N.A.; Eshraghi, A.; Gholizadeh, H.; Abd Razak, N.A. Bin; Wan Abas, W.A.B. Bin Interface pressure in transtibial socket during ascent and descent on stairs and its effect on patient satisfaction. Clin. Biomech. 2013, 28, 994-999, doi:10.1016/j.clinbiomech.2013.09.004.

36. Boutwell, E.; Stine, R.; Hansen, A.; Tucker, K.; Gard, S. Effect of prosthetic gel liner thickness on gait biomechanics and pressure distribution within the transtibial socket. J. Rehabil. Res. Dev. 2012, 49, 227-240, doi:10.1682/JRRD.2010.06.0121.

54

55

56 57

58

59

60

61

62

63

64

65

66 67 68 69 70 71 72 73 74 
1. Michael A. McGeehan is a post-doctoral scholar at the University of Oregon's Phil and Penny Knight Campus for Accelerating Scientific Impact. He received the M.S. degree in 2016 and the Ph.D. degree in 2020 from the University of Oregon's Department of Human Physiology with an emphasis in biomechanics. His research areas include computational modeling, simulations, and sensor development as they relate to orthopedic rehabilitation. Dr. McGeehan's overarching research goal is to apply principles of biomechanics, physiology, and engineering toward developing rehabilitation technology that improves the lives of people with disabling conditions.

2. Michael E. Hahn received the B.S. degree in biological sciences in 1996 from Colorado Mesa University, the M.S. degree in exercise and sports science in 2000 from Iowa State University, and the Ph.D. degree in exercise and movement science in 2003 from the University of Oregon. He served on the faculty of health and human development at Montana State University Bozeman for five years, then as Motion Laboratory Director in the VA Puget Sound in Seattle for four years, and is currently an Associate Professor in the department of human physiology and the Director of the Bowerman Sports Science Clinic at the University of Oregon. His research interests include injury prevention and performance prediction in athletes, and design of machine learning applications in control of lower limb prostheses. He is a member of the National Academy of Inventors, serves on the editorial boards of several musculoskeletal research journals, and is the author of over 60 peer-reviewed scientific publications.

3. Salil Sidharthan Karipott received his B. Tech. degree in Electronics and Biomedical Engineering from Cochin University of Science and Technology in 2014. He earned his M.S. and Ph.D. degrees from the Department of Biomedical Engineering at Michigan Technological University in 2017 and 2019, respectively. He is currently a post-doctoral researcher at the Knight Campus for Accelerating Scientific Impact, University of Oregon. His work focuses on developing wireless sensing and actuating devices for orthopedic research. He has expertise in developing implantable inductive-capacitive sensors, and implementing magnetoelastic actuators for studying mechanotransduction in bone fracture healing.

4. Keat Ghee Ong received his B.S., M.S., and Ph.D. degrees in Electrical Engineering from the University of Kentucky in 1997, 1998, and 2000, respectively. He joined the department of Biomedical Engineering at Michigan Technological University, Houghton, MI in 2006. In 2019, he joined the Knight Campus for Accelerating Scientific Impact at the University of Oregon as a professor. His research areas include implantable sensors for regenerative medicine and sensor technologies for cell manufacturing. He is also working on wireless sensor networks, magnetoelastic materials, and measurement technique and instrument automation. Ong is a senior member of National Academy of Inventors. He is on the editorial boards of multiple sensor related journals, and is the author of over 100 publications in archival journals. 